\title{
Editorial
}

Andrew P.J. Olson and Mark L. Graber

\section{Learning from tragedy - improving diagnosis through case reviews}

https://doi.org/10.1515/dx-2018-0068

In this issue of Diagnosis, we are initiating a new feature: “Case Reports of Diagnostic Error”. Many journals feature case reports, with the most well-known being the "Case Records of the Massachusetts General Hospital" that have appeared in the New England Journal of Medicine for most of the past century. These are addressed to health care professionals and meant to be instructional, focusing on the disease and its differential diagnosis, while also exploring the pathophysiology and clinical features. The purpose of the case reports published in Diagnosis is novel and completely different: the focus of these reports is not the disease, but the diagnostic process. The goal is learning how to improve that process, thereby avoiding harm related to diagnostic error for future patients. There is another unique feature of these reports that has been lacking in traditional reports: they will include the perspectives (as much as possible) of the patient or family, the providers involved in the patient's care and other major stakeholders. This is fundamental, as the hope of every family of every patient harmed by diagnostic error is that a similar error never happens to another person. Thus, sharing these stories is an important step in fulfilling these families' aching desires.

The first case in this series reports the tragic death of Julia Berg, a 15-year-old girl, who died after an unnecessary operation for presumed cholecystitis. The actual diagnosis, discovered post-mortem, was Epstein-Barr virus (EBV) infection with hepatitis, that is, mononucleosis. What can be learned from this case about improving diagnosis?

The first lesson is the value of learning from an indepth analysis of a single case. The diagnostic process is uniformly idiosyncratic, and a nuanced approach must be taken when analyzing the diagnostic process that unfolded in a given case. This case highlights the importance of skillfully navigating all the steps of the diagnostic process; it is conceivable, if not likely, that Julia may have survived her EBV infection if greater attention had been paid to her presenting complaints and laboratory findings. All the necessary facts were available to make the correct diagnosis, but because her history of fatigue was dismissed, and her striking lymphocytosis was not appreciated as being salient, the correct diagnosis was not considered. The team locked in on an 'obstructive' pattern of liver function tests, and interpreted this as representing a diseased gall bladder. Lymphocytosis would be uncommon in cholecystitis while it is a classic finding in EBV infection - in this case, the finding that did not fit really mattered. In case series of diagnostic error, these breakdowns in appreciating the salience of findings, and synthesizing the available information, are the most common elements contributing to error [1, 2]. There is great educational value in highlighting when findings don't fit - and encouraging health care professionals to ask "What if the finding that doesn't fit really matters?" with every patient.

The next lesson from this case is the value of the autopsy in revealing the correct diagnosis. Although autopsies have virtually disappeared in many centers, there are a few remaining centers interested in performing these procedures. In 2014, a specialty center in Europe reported a major diagnostic discrepancy rate of $23 \%$ [3], and a series of 334 cases from the United States found a 10\% discrepancy rate [4]. Thus even in the 'high tech' era, the autopsy has value in identifying diagnostic errors, and remains the gold standard as a research tool. Given these striking discrepancy rates, we believe it is a moral obligation to ensure that providers and families know the actual diagnosis affecting patients. Reviving the autopsy as a tool for learning was a major recommendation of the National Academy of Medicine Report on Improving Diagnosis in Health Care [5] that has yet to be acted upon. There are multiple reasons for autopsies not being performed, but these factors are completely surmountable and restoring an appreciable rate of autopsies is central to improving our understanding of our diagnostic outcomes. Autopsies are likely one of the most effective means of learning from harmful diagnostic (and other) errors and it is unacceptable to forego them for reasons of expediency.

A third lesson relates to the what can be learned (and what cannot be learned) about diagnosis from 
administrative data. There is a growing trend to study diagnostic outcomes from 'big data', that is, using administrative data derived from the electronic record and associated coding linked to each encounter. This approach has been used successfully, for example, to study diagnostic error associated with stroke [6]. Julia's case is a powerful reminder, however, of the limitations imposed by looking just at administrative data. Her death certificate lists the cause of death as fulminant EBV infection and recent cholecystectomy. Would a computer algorithm have interpreted these discordant two facts as representing a diagnostic error? In short, our current coding practices and the many inaccuracies captured on death certificates create serious limitations on the value of trying to learn from aggregated analyses that rely on this information [7]. It is likely that complex algorithms will need to be developed to search for "signals" that signify diagnostic error in these large datasets until a code that designates a case as a diagnostic error (or missed opportunity) is developed. This does not yet exist but would have substantial value for research and learning, not to mention transparency and culture change.

\section{Feedback is the key}

It is generally held that improving human performance - including diagnostic performance - occurs most effectively through an individual focusing on improving in specific areas and receiving targeted, regular feedback about their performance [8]. This deliberate practice is unfortunately rare in medicine, and in many cases practitioners don't know the outcomes of their decisions. Even most cases of diagnostic error are likely unknown to the provider who made the decisions lead to the error. Individuals must be insatiable in their curiosity after an adverse event occurs, and systems must support providers in learning about and from their outcomes. In our opinion, it is a moral and educational imperative to ensure that health care providers have an opportunity to learn from the outcomes of their decisions - especially in cases of diagnostic error - every single time.

There are many challenges to ensuring high-quality, universal feedback about medical decision-making, including both interpersonal and systemic challenges. Interpersonal barriers include ensuring psychological safety, fear of litigation, lack of patient "ownership", the balance between developing autonomy and instilling humility and avoiding a punitive, blaming environment. These interpersonal challenges are surmountable, however, when a health care system and especially clinical supervisors model discussions of errors as opportunities for learning $[9,10]$. Some systemic challenges include frequent transitions of care between providers and care settings, poor electronic health record (EHR) interoperability, and the lack of features in the EHR that might help promote better follow-up. The lack of time for this feedback to occur is oft discussed, yet this question must be asked: "How is it acceptable that we have time to make errors, but not enough time to learn from them?"

Despite these barriers, however, it is fundamental that health systems and medical educators begin to ensure that feedback loops are closed for as many cases as possible. Without that feedback, there is no incentive to learn. The American author and reporter Katherine Schulz asks the question: “What does it feel like to be wrong?” [11]. The surprising answer is: It feels exactly like we are right until we are proven to be wrong. Humans view unknown outcomes the same as they view positive outcomes - we are wired to be optimists and truly believe that "no news is good news".

If future performance is to be improved through education, we must ensure that nearly all outcomes are knowable and known by the decision makers who contribute to those outcomes. The paradigm must change: "No news is not good news, it is just no news". We owe it to our past, present and future patients to learn from and share their stories.

Author contributions: All the authors have accepted responsibility for the entire content of this submitted manuscript and approved submission.

Research funding: None declared.

Employment or leadership: None declared. Honorarium: None declared.

\section{References}

1. Schiff GD, Hasan O, Kim S, Abrams R, Cosby K, Lambert B, et al. Diagnostic error in medicine - analysis of 583 physician-reported errors. Arch Int Med 2009;169:1881-7.

2. Graber ML, Franklin N, Gordon R. Diagnostic error in internal medicine. Arch Intern Med 2005;165:1493-9.

3. Kuijpers C, Fronczek J, van de Goot F, Niessen H, van Diest P, Jiwa $M$. The value of autopsies in the era of high-tech medicine: discrepant findings persist. J Clin Pathol 2014;67:512-9.

4. Marshall H, Milikowski C. Comparison of clinical diagnosis and autopsy findings: six-year retrospective study. Arch Pathol Lab Med 2017;141:1262-6.

5. Institute of Medicine. Improving diagnosis in health care. Washington, DC: National Academies Press, 2015. 
6. Newman-Toker D, Moy E, Valente E, Coffey R, Hines AL. Missed diagnosis of stroke in the emergency department: a crosssectional analysis of a large population-based sample. Diagnosis 2014;1:155-66.

7. Makary M. Medical error - the third leading cause of death in the US. BMJ 2016;353:i2139. doi: 10.1136/bmj.i2139.

8. Ericsson K. Acquisition and maintenance of medical expertise: a perspective from the expert-performance approach with deliberate practice. Acad Med 2015;90:1471-86.

9. Ruedinger E, Mathews B, Olson A. Decision - Diagnosis: an introduction to diagnostic error and medical decision-making. MedEdPortal 2016. https://www.mededportal.org/publication/10378/.
10. Ruedinger E, Olson M, Yee J, Borman-Shoap E, Olson APJ. Education for the next frontier in patient safety: a longitudinal resident curriculum on diagnostic error. Am J Med Quality 2017;32:625-31.

11. Schulz K. Being wrong: adventures in the margin of error. NY, NY: Ecco - Harper Collins, 2010.

Corresponding author: Mark L. Graber, MD, FACP, 5 Hitching Post, Plymouth, MA 02360, USA,

E-mail: mark.graber@improvediagnosis.org

Andrew P.J. Olson: University of Minnesota Medical School, Departments of Medicine and Pediatrics, Minneapolis, MN, USA, E-mail: ols05714@umn.edu 